PASJ: Publ. Astron. Soc. Japan , 1-??,

(C) 2021. Astronomical Society of Japan.

\title{
Variable Selection for Modeling the Absolute Magnitude at Maximum of Type Ia Supernovae
}

\author{
Makoto Uemura, Koji, S. Kawabata \\ Hiroshima Astrophysical Science Center, Hiroshima University, Kagamiyama 1-3-1, Higashi-Hiroshima, 739-8526, Japan \\ Core of Research for the Energetic Universe, Hiroshima University, Higashi-Hiroshima, Hiroshima 739-8526, Japan \\ uemuram@hiroshima-u.ac.jp \\ Shiro IKEDA \\ The Institute of Statistical Mathematics and CREST, JST, Tachikawa, Tokyo, 190-8562, Japan \\ and \\ Keiichi MAEDA \\ Department of Astronomy, Kyoto University, Kitashirakawa-Oiwake-cho Sakyo-ku, Kyoto 606-8502, Japan \\ Kavli Institute for the Physics and Mathematics of the Universe (WPI), University of Tokyo, 5-1-5 Kashiwanoha, Kashiwa, \\ Chiba 277-8583, Japan
}

(Received ; accepted )

\begin{abstract}
We discuss what is an appropriate set of explanatory variables in order to predict the absolute magnitude at the maximum of Type Ia supernovae. In order to have a good prediction, the error for future data, which is called the "generalization error," should be small. We use cross-validation in order to control the generalization error and LASSO-type estimator in order to choose the set of variables. This approach can be used even in the case that the number of samples is smaller than the number of candidate variables. We studied the Berkeley supernova database with our approach. Candidates of the explanatory variables include normalized spectral data, variables about lines, and previously proposed flux-ratios, as well as the color and light-curve widths. As a result, we confirmed the past understanding about Type Ia supernova: i) The absolute magnitude at maximum depends on the color and light-curve width. ii) The light-curve width depends on the strength of Si II. Recent studies have suggested to add more variables in order to explain the absolute magnitude. However, our analysis does not support to add any other variables in order to have a better generalization error.
\end{abstract}

Key words: supernovae: general

\section{Introduction}

Type Ia supernovae (SNe Ia) have been used as "standard candles" to estimate the distance to galaxies in cosmology. Phillips (1993) found a significant correlation between their absolute magnitude at maximum, $M$, and decay rate, and proposed that a better distance indicator can be obtained by calibrating it. As well as the decay rate, the observed color also exhibits a clear correlation with $M$. This is mainly due to the interstellar extinction in both their host and our galaxies, while it is proposed that there is a variation in the intrinsic color of SNe Ia at maximum (Conley et al. 2007; Foley, Kasen 2011). In addition to these two, a number of variables have been proposed as explanatory variables of $M$. They are, for example, the equivalent widths, velocities, or depths of absorption lines, or their ratios (for a review, see Silverman et al. 2012).

The search for a good set of variables, in other words, the "model," have recently been intensified including arbitrary ratios of the fluxes in spectra. Using the 58 objects observed by Nearby Supernova Factory, Bailey et al. (2009) report that the model with a single ratio of the flux at $642 \mathrm{~nm}$ to that at $443 \mathrm{~nm}$, hereafter $\mathcal{R}(642 \mathrm{~nm} / 443 \mathrm{~nm})$, has a smaller residual of $M$ than the classical model with the color and decay rate (, or light-curve width). Using 26 objects observed by the CfA Supernova Program, Blondin et al. (2011) confirm the conclusion in Bailey et al. (2009) with a slightly different ratio, $\mathcal{R}(6630 \AA / 4400 \AA)$, although the improvement of the model has low significance. In addition, they propose another model with the color and the color-corrected flux ratio, $\mathcal{R}^{c}(4610 \AA / 4260 \AA)$ at $t=-2.5 \mathrm{~d}$ from maximum light. Silverman et al. (2012), using 62 object observed by the Berkeley Supernova Ia Program, report that the best set of variables is the light-curve width, color, and $\mathcal{R}^{c}(3780 \AA / 4580 \AA)$. On the other hand, their analysis did not confirm the results in Bailey et al. (2009) and Blondin et al. (2011). Thus, the resulting models of each work are not completely consistent, and the model for the prediction of $M$ has not been established.

In previous studies, a linear regression model of $M$ has been assumed:

$$
M_{B} \simeq M_{B, 0}+\beta_{1} x_{1}+\beta_{2} x_{2}+\cdots+\beta_{L} x_{L},
$$

where $M_{B}$ is the absolute magnitude in the $B$-band, which has been conventionally used in past studies. $M_{B, 0}$ is a constant. The vector, $\boldsymbol{x}=\left(x_{1}, x_{2}, \cdots, x_{L}\right)^{T}$ is a set of explanatory variables of $M_{B}$. The elements in $\boldsymbol{x}$ are, for example, the color, decay rate (, or light-curve width), and 
variables about the lines. $\boldsymbol{\beta}=\left(\beta_{1}, \beta_{2}, \cdots, \beta_{L}\right)^{T}$ is the vector of their coefficients. Suppose that $N$ samples of SNe Ia are available, and the observations are summarized as $\boldsymbol{y} \simeq X \boldsymbol{\beta}$, where $\boldsymbol{y}=\left(M_{B 1}, M_{B 2}, \cdots, M_{B N}\right)^{T}$ and $X=\left(\boldsymbol{x}_{1}, \cdots, \boldsymbol{x}_{N}\right)^{T}$. The goal of the study is to find an appropriate set of variables in $\boldsymbol{x}$ for the prediction of $\boldsymbol{y}$. We prefer the model to have a small generalization error for the prediction of $\boldsymbol{y}$.

If $N \geq L$, it is possible to estimate the values of all elements in $\boldsymbol{\beta}$ with the least-square method. However, the risk of over-fitting increases as $N / L$ becomes smaller. Furthermore, the least-square method cannot determine a unique model when $N<L$. Such a situation can appear when arbitrary flux ratios in spectra are included into $X$. Hence, previous studies included only one or two flux ratios in a model, and search for the best set of the variables for the observations.

Finding an appropriate set of variables to describe $M_{B}$ of SNe Ia is a variable selection problem, which has been studied in the field of statistics and machine learning. In this paper, we report a result of variable selection approach applied for $M_{B}$. We controlled the generalization error with a regularization term, whose size is chosen via cross-validation, and a subset of the variables are selected from $L$ components by Least Absolute Shrinkage and Selection Operator, or the so-called, LASSO method (Tibshirani 1996). This method can find the unique solution even in the case of $N<L$. In section 2 , we describe the method. In section 3 , we report on the results of our experiments. We apply our method to the data provided by the Berkeley supernova database. In section 4, we discuss the implication of our results, and summarize our findings.

\section{Method}

\subsection{LASSO-type estimation}

Here, we consider a linear regression model, $\boldsymbol{y}=X \boldsymbol{\beta}+\boldsymbol{e}$, where $X$ is a given real $N \times L$ matrix and $e$ is a Gaussian noise with $E[\boldsymbol{e}]=\mathbf{0}$ and $E\left[\boldsymbol{e} \boldsymbol{e}^{\prime}\right]=\sigma^{2} I_{N}$. Our goal is to find an appropriate set of variables from $L$ variables and $N$ samples and compute the corresponding coefficients of $\boldsymbol{\beta}$. For this sort of estimation problems, Tibshirani (1996) proposed a method, Least Absolute Shrinkage and Selection Operator, or the so-called, LASSO, for selecting the best set of explanatory variables. LASSO provides a solution $\hat{\boldsymbol{\beta}}$ by minimizing the following function which includes the $\ell 1$-norm of $\boldsymbol{\beta}$ as a regularization term

$$
\hat{\boldsymbol{\beta}}_{\lambda}=\underset{\boldsymbol{\beta}}{\arg \min }\left\{\|\boldsymbol{y}-X \boldsymbol{\beta}\|_{2}^{2}+\lambda\|\boldsymbol{\beta}\|_{1}\right\},
$$

where $\|\boldsymbol{\beta}\|_{1}$ is the $\ell$ 1-norm, defined as $\|\boldsymbol{\beta}\|_{1}=\sum_{i}\left|\beta_{i}\right|$, and $\lambda$ is a tunable constant. The estimate $\hat{\boldsymbol{\beta}}$ includes 0 components, that is, variables selection is realized with LASSOtype estimation. The number of 0 components increases as $\lambda$ becomes larger.

We apply the LASSO-type estimation in order to select an appropriate model to predict $M_{B}$ of SNe Ia. The data, $\boldsymbol{y}$, is $M_{B}$ and each column of $X$ corresponds to an ob- served variables, such as, the color, light-curve width, and variables about spectra. Recent projects have provided high-quality and uniform samples of SNe Ia in both photometric and spectroscopic data. The number of available samples, $N$, is now $\sim 100$. The number of candidate explanatory variables can be $>10^{4}$ if arbitrary flux ratios are included. However, we can expect that the number of effective variables is small. In other words, our interest focuses on a model in which $M_{B}$ is explained not with $\sim 10^{4}$, but with only a few variables of $\boldsymbol{x}$. Exhaustive search for every subset of candidate variables is not tractable, and the LASSO-type estimation gives us a data-driven approach to select the best subset of variables for the dataset.

\subsection{Cross-validation}

The cost function for the estimation expressed in equation (2) contains a tunable parameter, $\lambda$. This parameter controls the weight of the regularization term, which has an influence on the generalization error. We choose the best $\lambda$ by the cross-validation method. In the $K$-fold cross-validation, the data is divided into $K$ roughly equal sub-samples, $\boldsymbol{y}_{k}(k=1,2, \cdots, K)$. For each $k$, the training data is defined as all the $K-1$ sub-samples except for the validation data, $\boldsymbol{y}_{k}$. The optimization of the model to the training data gives $\hat{\boldsymbol{\beta}}_{k, \lambda}$ at a certain $\lambda$. The generalization error of the model is evaluated with the mean of weighted mean square errors (wMSE; $E(\lambda)$ ) of the $K$ sub-samples;

$$
\begin{aligned}
E(\lambda) & =\frac{1}{K} \sum_{k=1}^{K} E_{k}(\lambda) \\
E_{k}(\lambda) & =\frac{\sum_{i=1}^{M_{k}}\left(y_{k, i}-\hat{y}_{k, \lambda, i}\right)^{2} / \sigma_{k, i}^{2}}{\sum_{i=1}^{M_{k}} 1 / \sigma_{k, i}^{2}} \\
\hat{y}_{k, \lambda, i} & =\sum_{j=1}^{N} x_{i, j} \hat{\beta}_{k, \lambda, j}
\end{aligned}
$$

where $M_{k}$ is the number of the validation data, $\boldsymbol{y}_{k}$, and $\sigma_{k, i}$ is the measurement error of the $i$-th element in $\boldsymbol{y}_{k}$.

In a very large $\lambda$ regime, the least-square term is large, and thereby $E(\lambda)$ also becomes large. In a very small $\lambda$ regime, on the other hand, the model can reproduce the noise in the data (over-fitting), and thereby have a large generalization error, and eventually lead to a large $E(\lambda)$. Thus, we can find the minimum value of $E(\lambda)$ at a certain $\lambda$. The best model can be considered as the simplest model whose $E(\lambda)$ is within one standard error of the minimal $E(\lambda)$. This is the so-called "one standard error rule". Models having $\lambda$ smaller than the best one are statistically indistinguishable from the over-fitting situation. In this paper, we use this rule to select $\lambda$, and set $K=10$.

Another common variable selection scheme is to use an information criterion, such as Akaike information criterion (AIC) or Bayesian information criterion (BIC). We employed the regularization term and the cross-validation because we expect not only the observation noise $\boldsymbol{e}$ but also the measurement errors in $X$, and we do not have a good model selection criterion for this situation. The 
measurement error of $M_{B}$ is occasionally quite small, an order of $0.01 \mathrm{mag}$. On the other hand, the error of the elements in $X$ can be large. For example, a ratio between low fluxes can have a large error.

\subsection{Demonstration of the method}

We performed simple simulations of the LASSO-type estimation for the current problem. The vector, $\boldsymbol{\beta}$, was set to be a sparse vector, containing only three non-zero values in $L$ elements. We set three cases: $L=10^{2}, 10^{3}$, and $10^{4}$. The matrix, $X$, was set to be a $N \times L$ matrix whose elements were random values generated by $\mathcal{N}(0,1)$, a normal distribution with a mean of 0 and variance of 1 . We set $N=50$ in all cases. Then, we calculated the data vector, $X \boldsymbol{\beta}$, and added noise, $\boldsymbol{y}=X \boldsymbol{\beta}+\boldsymbol{e}$, $\boldsymbol{e} \sim \mathcal{N}\left(\mathbf{0}, 0.01 \sigma_{\boldsymbol{y}}^{2} I_{N}\right)$, where $\sigma_{\boldsymbol{y}}$ represents the standard deviation of observation noise. Here, we assumed a small error in $\boldsymbol{y}$ because $M_{B}$ is occasionally determined with such a high precision. We also added noise in the elements of $X, \tilde{x}_{i j}=x_{i j}+\epsilon_{i j}, \epsilon_{i j} \sim \mathcal{N}\left(0, \sigma_{X}^{2}\right)$, and generated $\tilde{X}$. We assumed small and large errors in $X$, that is, $\sigma_{X}=0.01$ and 0.25 . We estimated $\boldsymbol{\beta}$ from $\boldsymbol{y}$ and $\tilde{X}$ using the $\ell 1$-norm minimization. The best model and its $\lambda$ were determined by cross-validation. The results are shown in figure 1 . In the case of the small $\sigma_{X}$, the assumed $\boldsymbol{\beta}$, indicated by the red points, are successfully reconstructed in all $L$ cases, albeit with a $3-20 \%$ systematic bias. In the case of the large $\sigma_{X}$, all non-zero elements in $\boldsymbol{\beta}$ are detected in the cases of $L=10^{2}$ and $10^{3}$, while their coefficients are significantly underestimated and weak false signals are also seen. In the case of $L=10^{4}$, the assumed weak signal is lost in the reconstruction, and false signals have large coefficients.

This experiment demonstrated two important points about the proposed method. First, it can reconstruct the original vector even with the case of $N<L$. Second, even with this method, we cannot avoid detecting false signals which are coincidentally fit the data in the case of a large $L$. The latter point could have a significant implication for using the arbitrary flux ratios in the current problem. The number of the flux ratios is more than 17000 , while the number of samples is $\lesssim 100$. Hence, we should reduce the number of columns in $X$ in order to avoid detecting the false signals. In this paper, as described in the next subsection, we use two kinds of spectra normalized by the continuum level and by the total flux.

LASSO tends to underestimate the coefficients if the measurement error of the target variable is not negligible, as can be seen in figure 1 . Hence, it should be used to select the best set of variables. Then, the model of $M_{B}$ can be obtained by a refit to the data with the selected variables. In this paper, we focus on variable selection.

\subsection{Sample and variables}

We used the data from SuperNova DataBase provided by Berkeley Supernova Ia program ${ }^{1}$. Our sample selection was based on the criteria in Silverman et al. (2012):

1 〈http://hercules.berkeley.edu/database/index_public.html $\rangle$
The redshift of the sample ranged from 0.01 to 0.1 . We used the spectral data from 3500 to $8500 \AA$. The restframe days relative to the maximum is ranged from -5 to $+5 \mathrm{~d}$. We used the spectrum having the smallest value of the rest-frame days relative to the maximum for each object in the case that multiple spectra were available. We only used samples having the color parameter, $c$, less than 0.5. We found two Type Iax objects, SN 2003gq and 2005hk in the sample, and excluded them (Foley et al. 2013). As a result, we found 78 objects in the database. The available data contains, for example, the redshift, $z$, light-curve width, $x_{1}$, color, $c$, apparent magnitude, $m_{B}$, and spectra. As mentioned in section 1, it is believed that $x_{1}$ and $c$ are important explanatory variables for $M_{B}$. We calculated $M_{B}$ from $m_{B}$ and $z$ by adopting the standard $\Lambda$ cold dark matter cosmology with $\Omega_{m}=0.27, \Omega_{\Lambda}=0.73$, and $w=-1$.

The calibration of the spectral data was performed in the standard manner: The flux was corrected for the reddening in our galaxy using $E(B-V)$. We used the $E(B-V)$ values obtained from the supernova database, which refers to Schlegel et al. (1998) and Peek, Graves (2010). The red-shift correction was performed on the wavelength. Then, the spectra were divided into 134 bins which were equally spaced in the logarithmic velocity scale between 3500 and $8500 \AA$, as in Silverman et al. (2012). We calculated the arbitrary flux ratios using the binned spectra. The number of the ratios is then $134 \times 133=17822$.

Including arbitrary flux ratios may provide an exhaustive search for an appropriate set of explanatory variables of $M_{B}$. However, the number of candidate variables is so large that false signals can be detected, as demonstrated in the last subsection. Hence, we need to consider other sets of candidate variables which are related to the flux ratios, but have much smaller dimension. In this paper, we use two kinds of normalized spectra.

First, the variables of the most interest are the flux ratios of the line areas to the continuum level. Indeed, most of previously proposed ratios are such variables: $\mathcal{R}(6420 / 4430)=$ Fe II/continuum (Bailey et al. 2009), $\mathcal{R}(6630 / 4400)=$ Fe II $/$ continuum, $\mathcal{R}(6420 / 5290)=$ contin uum/S II, and $\mathcal{R}(4610 / 4260)=$ continuum/Fe II (Blondin et al. 2011). They can be substituted by the spectra normalized by the continuum level. The continuum level was approximated by a cubic smoothing spline fitted to masked spectra. The mask is depicted in figure 2 with the binned spectra of a typical sample, SN 2006et. The data points indicated by the filled circles were used to calculate the continuum curve. In addition, the points with the maximum flux in each shaded area were also used. The several examples of the continuum-normalized spectra are shown in the lower panel of figure 3 . We call the set of the continuum-normalized spectra as $f_{\text {cnt }}$.

Second, the local colors in the continuum which may have independent information of the broadband colors are also variables of interest. They can be substituted by the spectra normalized by the total flux between 3500 and $8500 \AA$ A . We call the set of this total flux normalized spec- 

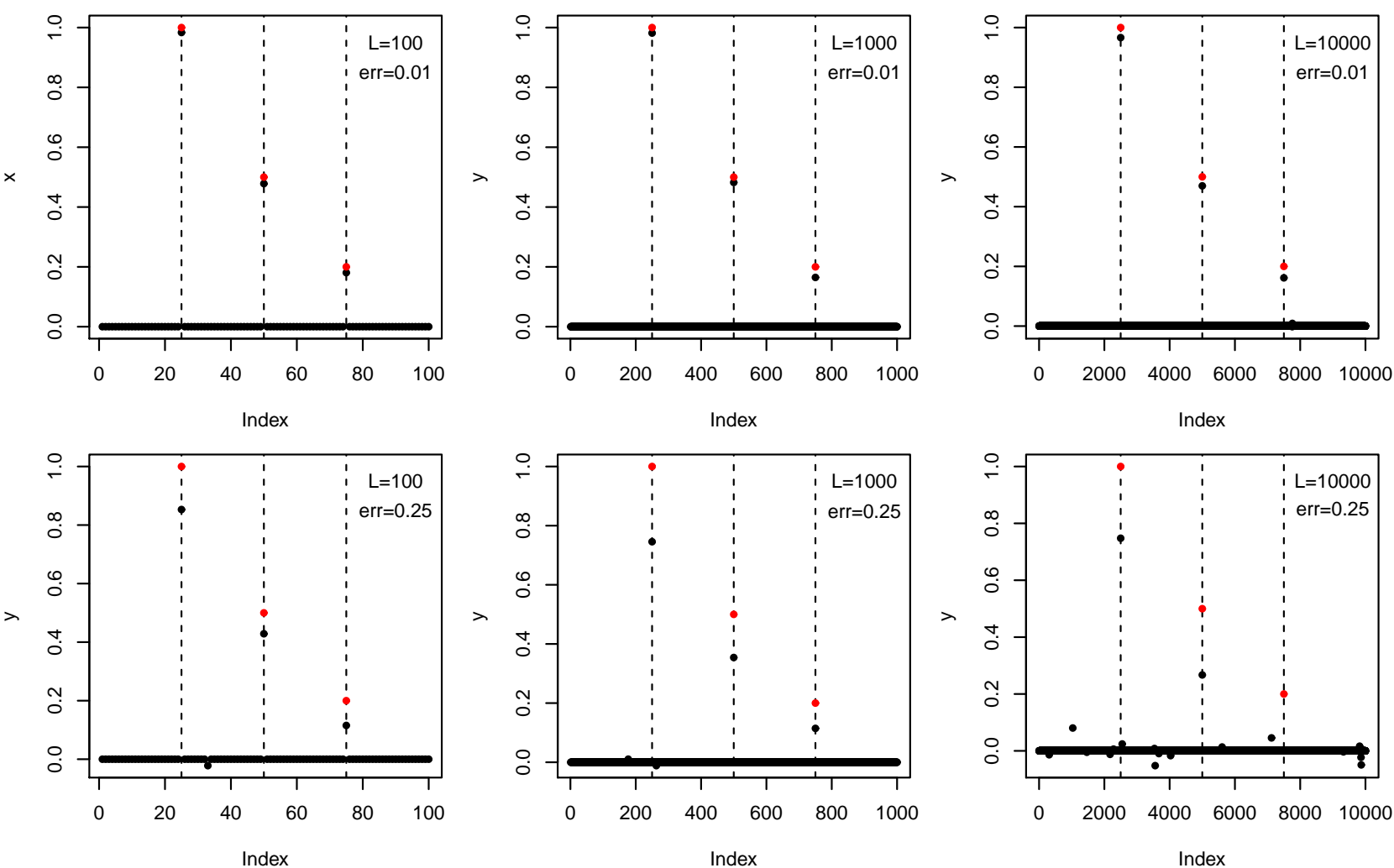

Fig. 1. Simulations of the LASSO-type estimation. The red and black points represent the assumed and estimated values, respectively. The number of samples is 50. The numbers of explanatory variables are $L=10^{2}$ (left), $10^{3}$ (middle), and $10^{4}$ (right), as shown in each panel. The upper and lower panels depict the cases for small and large errors assumed in $X$.

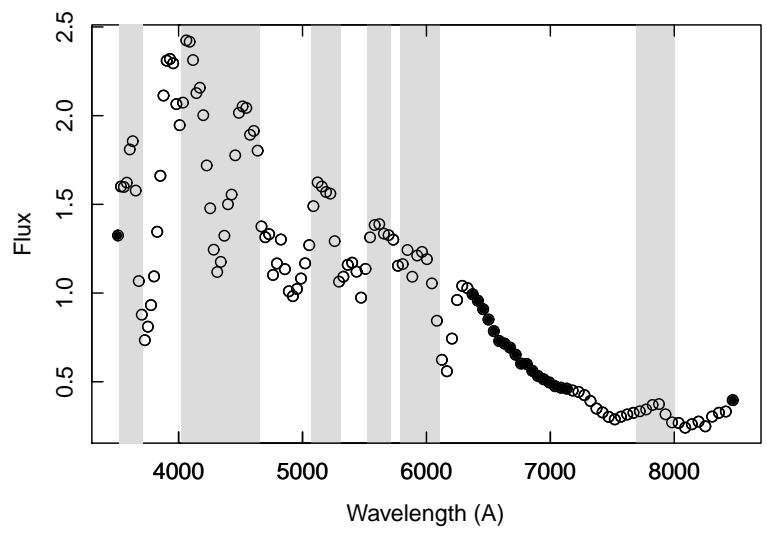

Fig. 2. Mask for calculating the continuum level. The spectrum of SN 2006et is also plotted as a reference. For detail, see the text.

tra as $\boldsymbol{f}_{\text {tot }}$. The intrinsic color can be bluer than the observed one because of the interstellar reddening effect in the host galaxy. In previous studies, the color correction for this effect has been performed by assuming that all $\mathrm{SNe}$ Ia have the same intrinsic color. We also performed this correction using the SNe Ia color-law for the SALT2 data (Guy et al. 2007). The color-corrected spectra is, then normalized by the total flux, and named $\boldsymbol{f}_{\text {tot }}^{c}$.
We include those two kinds of normalized spectra, $\left(\boldsymbol{f}_{\text {cnt }}, \boldsymbol{f}_{\text {tot }}\right)$ or $\left(\boldsymbol{f}_{\text {cnt }}, \boldsymbol{f}_{\text {tot }}^{c}\right)$ as the candidates, instead of the arbitrary flux ratios. In addition, we use the flux in the logarithmic scale in order to include the information of arbitrary flux ratios. We can identify a good flux-ratio parameter by searching for the two fluxes having the similar coefficients with the opposite sign: $c \cdot \log \left(f_{1} / f_{2}\right)=$ $c \cdot \log \left(f_{1}\right)-c \cdot \log \left(f_{2}\right)$. Figure 3 shows examples of the spectra that are normalized by the total flux $\left(f_{\text {tot }}\right.$, the upper panel) and by the continuum ( $\boldsymbol{f}_{\mathrm{cnt}}$, the lower panel).

As well as $x_{1}, c, \boldsymbol{f}_{\text {cnt }}, \boldsymbol{f}_{\text {tot }}$, and $\boldsymbol{f}_{\text {tot }}^{c}$, we include previously proposed flux-ratios, $\mathcal{R}$ into the model as candidate explanatory variables for $M_{B}$. We consider six flux-ratios proposed in Bailey et al. (2009), Blondin et al. 2011, and Silverman et al. (2012), that is, $\mathcal{R}=\{\mathcal{R}(3780 / 4580), \mathcal{R}(4610 / 4260), \mathcal{R}(5690 / 5360)$, $\mathcal{R}(6420 / 4430), \mathcal{R}(6420 / 5290), \mathcal{R}(6630 / 4400)\}$. The flux ratios which are calculated from the color-corrected spectra, $\boldsymbol{f}_{\text {tot }}^{c}$, are called as $\boldsymbol{\mathcal { R }}^{c}$.

Silverman et al. (2012) presents tables of measured values of the lines: Ca II H\&K and near-infrared triplet, Si II 4000, 5972, and $6355 \AA, \mathrm{Mg}$ II, Fe II, S II "W," and O I triplet. We can use pEW, Delta pEW (i.e., the measured pEW subtracted by the template evolution), velocity $(v)$, line depth $(a)$, and FWHM for the explanatory variables. We note that those line variables are incomplete for our sample. Hence, the number of samples reduces when those 

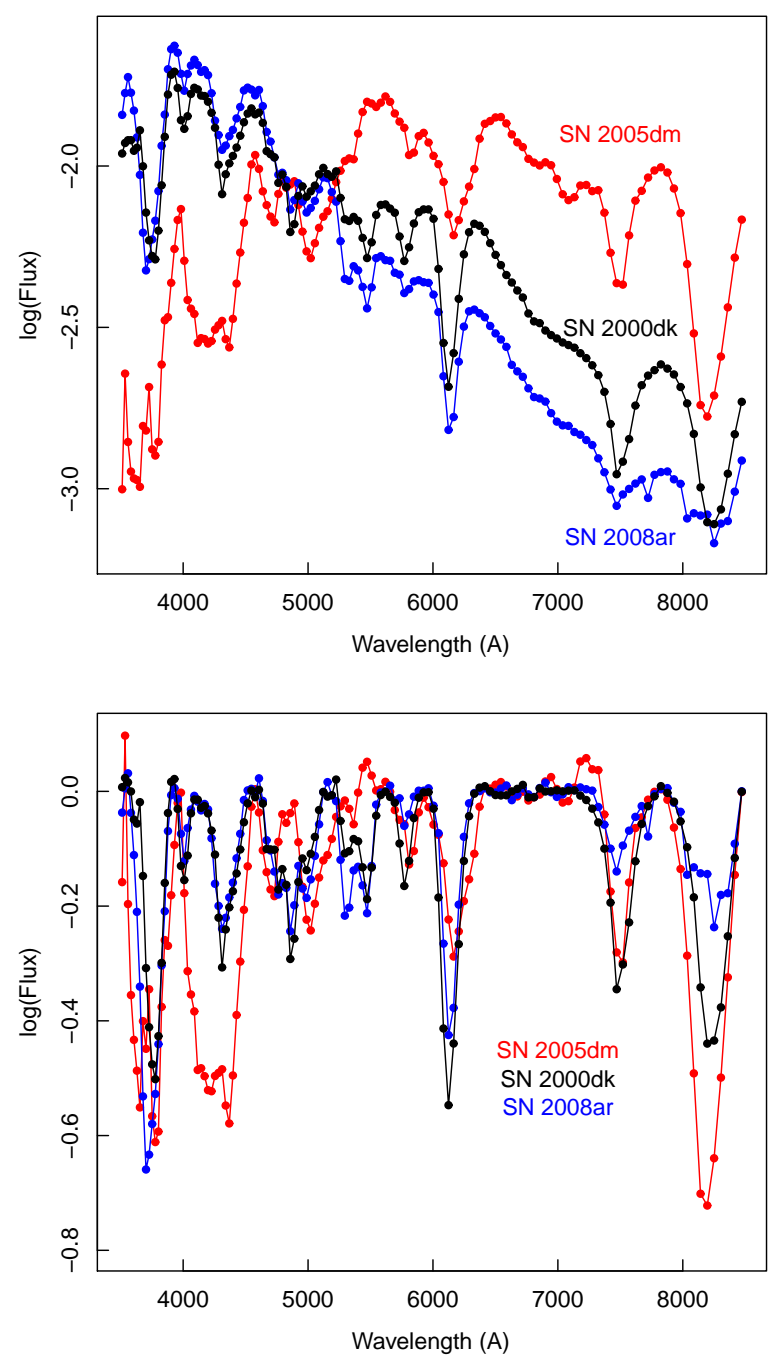

Fig. 3. Examples of the spectra in our sample. Upper panel: the spectra normalized by the total flux between $3500-8500 \AA$. Lower panel: the spectra normalized by the continuum. The spectra of three examples, SN 2005dm, SN 2000dk, and SN 2008ar, are shown in the both panels.

line variables are used as the candidate variables, and we used them element by element. We represent a set of the line values as $\mathcal{L}$. For example, $\mathcal{L}_{\text {Si II } 4000}$ means those variables of Si II $4000 \AA$.

For the optimization of the model to the data, we used the glmnet package for R. ${ }^{2}$ The selection of $\lambda$ was performed using the function for the cross-validation, cv.glmnet, adopting the one-standard error rule. The cross-validation is based on random sub-sampling and the selected variables might be influenced by it. We performed $10^{4}$ experiments for each model, and calculated the selection probability, $p$, of each variable. In this paper, we discuss selected variables only with $p>0.3$. Each column in $X$ was normalized to have zero mean and unit variance, by a linear scaling, $x_{i j}^{\prime}=\left(x_{i j}-\bar{x}_{j}\right) / \sigma_{j}$, where $\bar{x}_{j}$ and $\sigma_{j}$

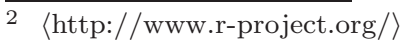

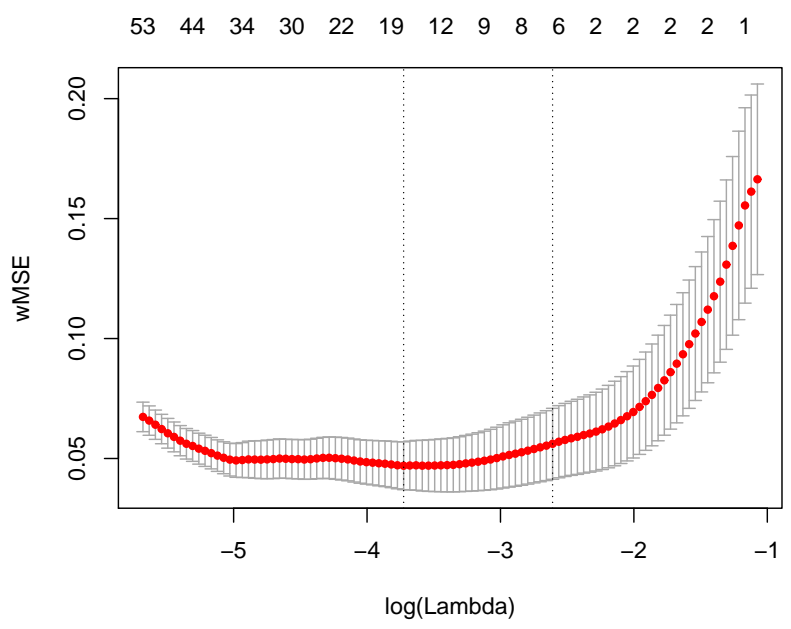

Fig. 4. Cross-validation curve for Model 1. The lower and upper horizontal axes denote $\lambda$ and the number of non-zero elements, respectively. The vertical axis denotes wMSE. The left dotted line indicates $\lambda$ having the minimal wMSE. The right dotted line indicates the best model under the one-standard error rule.

are the mean and standard deviation of the $j$-th column. We need this normalization to compare the coefficients, $\boldsymbol{\beta}$, of variables having different units. The list of objects and explanatory variables used in this paper is available as an online supplement material.

\section{Results}

First, we choose the light curve width $\left(x_{1}\right)$, color $(c)$, spectra normalized by the total flux $\left(\boldsymbol{f}_{\text {tot }}\right)$, those by the continuum $\left(\boldsymbol{f}_{\text {cnt }}\right)$, and previously proposed flux-ratios $(\mathcal{R})$ as the candidate explanatory variables, and $M_{B}$ as the target variable. We call this complete model as Model 1. It can be rewritten as:

$$
\begin{aligned}
M_{B} & =M_{B, 0}+\beta_{1} c+\beta_{2} x_{1} \\
& +\beta_{3} f_{\mathrm{tot}}(3512)+\beta_{4} f_{\mathrm{tot}}(3534)+\cdots+\beta_{136} f_{\mathrm{tot}}(8472) \\
& +\beta_{137} f_{\mathrm{cnt}}(3512)+\beta_{138} f_{\mathrm{cnt}}(3534)+\cdots+\beta_{270} f_{\mathrm{cnt}}(8472) \\
& +\beta_{271} \mathcal{R}(3780 / 4580)+\beta_{272} \mathcal{R}(4610 / 4260) \\
& +\beta_{273} \mathcal{R}(5690 / 5360)+\beta_{274} \mathcal{R}(6420 / 4430) \\
& +\beta_{275} \mathcal{R}(6420 / 5290)+\beta_{276} \mathcal{R}(6630 / 4400)+e .
\end{aligned}
$$

Using LASSO-type method for 78 samples of $M_{B}$, we choose the appropriate set of explanatory variables from 276 candidates and estimate coefficients vector $\boldsymbol{\beta}$. The tuning parameter, $\lambda$, is determined by cross-validation. Figure 4 shows the cross-validation curve for Model 1 . In this figure, we can confirm that wMSE take the minimum value in a given range of $\lambda$, and the best model is properly determined by the one-standard-error rule.

Table 1 lists all models and results presented in this paper. From Model 1, the classical variables, that is, $c$ and $x_{1}$ are selected. $f_{\text {tot }}(6373)$ is also selected, having a coefficient even larger than that of $x_{1}$ in the absolute val- 
Table 1. Models and Results

\begin{tabular}{|c|c|c|c|c|c|}
\hline Model & $\begin{array}{r}\text { Target variable } \\
\boldsymbol{y}(N)\end{array}$ & $\begin{array}{r}\text { Explanatory variables } \\
X(L)\end{array}$ & Non-zero elements & $\begin{array}{r}\text { coefficients } \\
\boldsymbol{\beta}\end{array}$ & $p$ \\
\hline \multirow[t]{8}{*}{1} & $M_{B}(78)$ & $x_{1}, c, \boldsymbol{f}_{\text {tot }}, \boldsymbol{f}_{\mathrm{cnt}}, \boldsymbol{\mathcal { R }}(276)$ & $c$ & 0.376 & 1.00 \\
\hline & & & $f_{\text {tot }}(6373)$ & 0.100 & 1.00 \\
\hline & & & $x_{1}$ & -0.050 & 0.98 \\
\hline & & & $f_{\text {cnt }}(6084)$ & -0.034 & 0.98 \\
\hline & & & $f_{\text {cnt }}(6289)$ & -0.045 & 0.95 \\
\hline & & & $f_{\text {cnt }}(6631)$ & -0.061 & 0.80 \\
\hline & & & $\mathcal{R}(3780 / 4580)$ & -0.050 & 0.74 \\
\hline & & & $f_{\text {tot }}(3752)$ & 0.063 & 0.73 \\
\hline 2 & $M_{B}-\beta_{1} c(78)$ & $x_{1}, \boldsymbol{f}_{\mathrm{tot}}, \boldsymbol{f}_{\mathrm{cnt}}, \mathcal{R}(275)$ & $x_{1}$ & -0.020 & 0.99 \\
\hline 3 & $M_{B}-\beta_{1} c(78)$ & $x_{1}, \boldsymbol{f}_{\mathrm{tot}}^{c}, \boldsymbol{f}_{\mathrm{cnt}}, \boldsymbol{\mathcal { R }}^{c}(275)$ & $x_{1}$ & -0.014 & 0.85 \\
\hline \multirow[t]{6}{*}{$4 \mathrm{a}$} & $x_{1}(76)$ & $c, \boldsymbol{f}_{\mathrm{tot}}^{c}, \boldsymbol{f}_{\mathrm{cnt}}, \mathcal{R}^{c}, \mathcal{L}_{\mathrm{Si} \text { II } 4000}(280)$ & $\mathrm{DpEW}_{\mathrm{Si} I \mathrm{I} 4000}$ & -0.455 & 1.00 \\
\hline & & & $f_{\text {cnt }}(5770)$ & 0.518 & 1.00 \\
\hline & & & $f_{\text {cnt }}(3982)$ & -0.262 & 1.00 \\
\hline & & & $f_{\text {cnt }}(7038)$ & -0.485 & 0.96 \\
\hline & & & $f_{\text {tot }}^{c}(4988)$ & -0.238 & 0.77 \\
\hline & & & $f_{\text {cnt }}(6084)$ & 0.281 & 0.62 \\
\hline \multirow[t]{7}{*}{$4 \mathrm{~b}$} & $x_{1}(74)$ & $c, \boldsymbol{f}_{\text {tot }}^{c}, \boldsymbol{f}_{\text {cnt }}, \mathcal{R}^{c}, \mathcal{L}_{\mathrm{SII}}$ "W" $(280)$ & $f_{\text {cnt }}(5770)$ & 1.034 & 1.00 \\
\hline & & & $f_{\text {cnt }}(6084)$ & 0.440 & 1.00 \\
\hline & & & $f_{\mathrm{tot}}^{c}(6458)$ & 0.300 & 1.00 \\
\hline & & & $f_{\text {cnt }}(3982)$ & 0.041 & 1.00 \\
\hline & & & $f_{\text {cnt }}(7179)$ & 0.289 & 0.99 \\
\hline & & & $f_{\text {cnt }}(6458)$ & -0.236 & 0.94 \\
\hline & & & $f_{\text {cnt }}(6331)$ & 0.612 & 0.92 \\
\hline
\end{tabular}

ues. Figure 5 indicates the non-zero elements of $\boldsymbol{f}_{\text {tot }}$ and $\boldsymbol{f}_{\text {cnt }}$. As can be seen in this figure, $f_{\text {tot }}(6373)$, indicated by the red vertical line, lies in the continuum area. Hence, it may be related to the local color which could have specific information against the broad-band color, $c$. As can be seen in figure 4 , some fluxes in line regions are also selected: $f_{\text {cnt }}(6084)$ and $f_{\text {cnt }}(6289)$ are probably related to the continuum-normalized depths of Si II(6355). In addition, $\mathcal{R}(3780 / 4580)$ and $f_{\text {tot }}(3752)$ are probably relate to Ca II H\&K. $f_{\text {cnt }}(6631)$ corresponds to the continuum flux of the continuum-normalized spectra, which suggests a false signal.

We confirmed that the result was consistent even when we included the line variables, $\mathcal{L}$, and all the line variables have zero coefficients in any elements. The lack of the dependency on $\mathcal{L}$ is common in other subsequent models, except for Model 4 (see below). Hence, we present the models only without $\mathcal{L}$ in this paper.

In general, when some explanatory variables are correlated, LASSO could select a few of them. In the present case, the variables are measurements having nonnegligible errors. Among correlated variables, a variable having a smaller error results in a smaller generalization error of $M_{B}$. Hence, in the case of large $\lambda$, the variable having the smallest error is first selected. In the case of smaller $\lambda$, the other correlated variables are selected. It is possible that a high correlation between $c$ and $f_{\text {tot }}(6373)$ would cause the non-zero coefficient of $f_{\text {tot }}(6373)$ in Model 1. If this is the case, it is unclear that

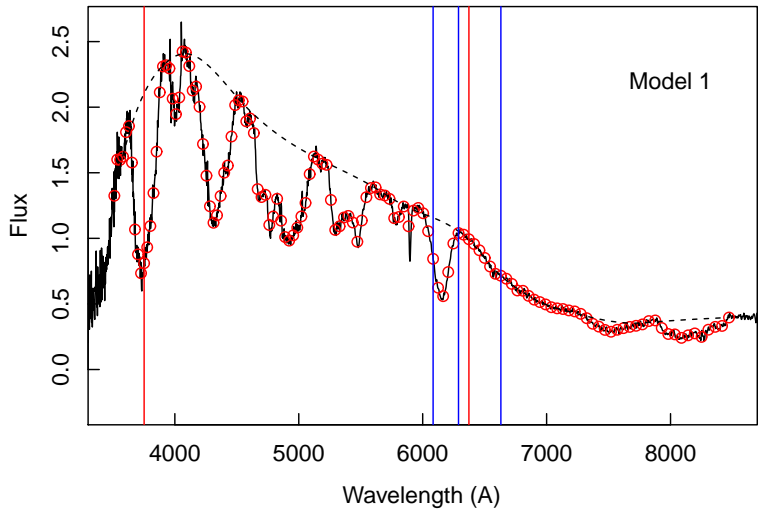

Fig. 5. Non-zero elements of spectral data in Model 1. The red and blue lines indicate the non-zero elements in the total-flux-normalized and continuum-normalized spectra, respectively. The spectrum of SN 2006et is also plotted as a reference. The solid and dashed lines and red points are unbinned spectra, estimated continuum level, and binned spectra, respectively. 

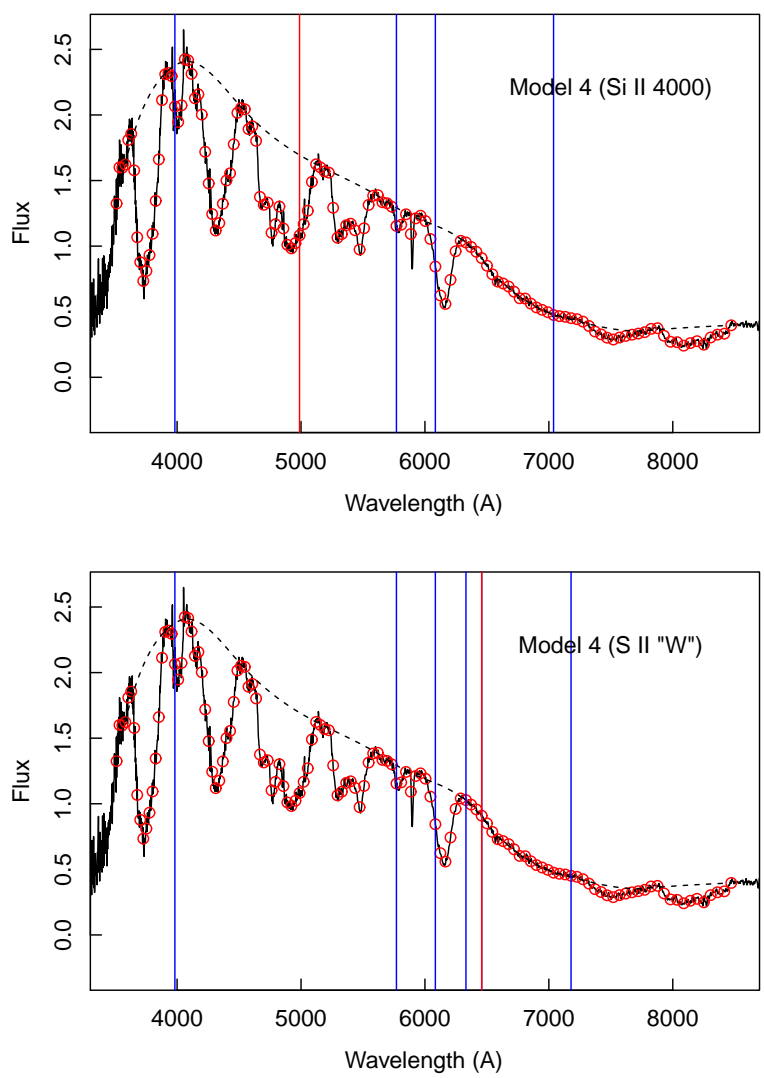

Fig. 6. Same as figure 5, but for Models $4 \mathrm{a}$ and $4 \mathrm{~b}$.

$f_{\text {tot }}(6373)$ is a significant variable that has independent information of $c$. We performed a regression analysis with $M_{B}=\beta_{1} c+M_{B, 0}$, and corrected for the effect of $c$ in $M_{B}$ by using $M_{B}-\beta_{1} c$ as the target. We call this complete model as Model 2. The number of samples is the same as that of Model 1, 78, while the number of candidate explanatory variables is 275 , one smaller than in Model 1 because $c$ is omitted. As can be seen in table $1, x_{1}$ is the only variable having a non-zero coefficient. A similar result was obtained for Model 3, where we used the color-corrected spectral data, $\boldsymbol{f}_{\text {tot }}^{c}$ and $\mathcal{R}^{c}$ instead of $\boldsymbol{f}_{\text {tot }}$ and $\mathcal{R}$. Hence, the lack of $f_{\text {tot }}(6373)$ is independent of the color correction. These results suggest that the high correlation between $c$ and $f_{\text {tot }}(6373)$ causes the apparently high coefficient of $f_{\text {tot }}(6373)$ in Model 1.

As well as $f_{\text {tot }}(6373)$, Model 1 indicates possible dependency of $M_{B}$ on the variables related to the line areas, that is, $f_{\text {cnt }}(6084)$ and $f_{\text {cnt }}(6289)$. It has been reported that $x_{1}$ depends on the line strength, for example, the EW of Si II 4000 (e.g. Hachinger et al. 2006; Arsenijevic et al. 2008). It is possible that the line dependency in Model 1 may be due to a high correlation between $x_{1}$ and the line strength of SiII. For examining this possibility, we considered Model 4 in which the target is $x_{1}$. Model $4 \mathrm{a}$ includes pEW, DpEW, $v, a$, FWHM of Si II 4000 , as well as $c, \boldsymbol{f}_{\text {tot }}^{c}, \boldsymbol{f}_{\text {cnt }}$, and $\boldsymbol{\mathcal { R }}^{c}$ as the candidate explanatory variables of $x_{1}$. This is the unique case where the coefficients of $\mathcal{L}$ have non-zero values in the analysis presented in this paper. Model $4 \mathrm{~b}$ is for S II "W", as a typical case of the other lines. The results are shown in table 1 and figure 6. In Model 4a, DpEW of Si II 4000 has a non-zero coefficient. The importance of this line is also confirmed by the selection of $f_{\text {cnt }}(3982)$ both in Models $4 \mathrm{a}$ and $\mathrm{b}$. In addition to Si II $4000, f_{\text {cnt }}(5770)$ and $f_{\text {cnt }}(6084)$ have non-zero coefficients in both models, corresponding to Si II 5972 and 6355. There are several other non-zero elements in Model 4a, although they are not confirmed in Model 4b. The dependence of $x_{1}$ on Si II supports the previous studies about $x_{1}$.

Finally, we employed Model 5, in which the target is $M_{B}$ corrected for $c$ and $x_{1}$, that is, $M_{B}-\left(\beta_{1} c+\beta_{2} x_{1}\right)$, where $\beta_{1}$ and $\beta_{2}$ are determined by a regression analysis. The candidate explanatory variables of Model 5 are $\boldsymbol{f}_{\text {tot }}^{c}$, $\boldsymbol{f}_{\text {cnt }}$, and $\boldsymbol{\mathcal { R }}^{c}$. However, any of them is not selected. The result suggests that the high correlation between $x_{1}$ and the Si II line strength results in the apparent dependency of $M_{B}$ on the line depths in Model 1. Hence, the best set of explanatory variables is $\left(c, x_{1}\right)$ in our analysis. We re-fit the data with these variables, and obtained the following model:

$$
M_{B}=-19.26( \pm 0.03)+2.75( \pm 0.17) c-0.10( \pm 0.02) x(7)
$$

Note that these values are calculated not from normalized values of the variables as in table 1, but from raw values.

\section{Discussion and Conclusion}

Our analysis confirms the classical understanding of $\mathrm{SNe}$ Ia, that is, i) the light-curve width $\left(x_{1}\right)$ and color (c) are the important explanatory variables of the absolute magnitude at maximum $\left(M_{B}\right)$ (Phillips 1993), and ii) the light-curve width correlates with the strength (EW or depth) of Si II (e.g. Hachinger et al. 2006; Arsenijevic et al. 2008). Furthermore, our variable selection approach using the LASSO-type estimation does not support to add any other variables, such as the normalized spectra $\left(f_{\text {tot }}\right.$, $\left.\boldsymbol{f}_{\text {tot }}^{c}, \boldsymbol{f}_{\text {cnt }}\right)$, previously proposed flux ratios $(\mathcal{R})$, and line measurements $(\mathcal{L})$, in order to have a better generalization error of $M_{B}$. We confirmed that the above conclusion is robust to small changes in our analysis: using the flux in logarithmic or linear scale, excluding or including two Type Iax objects, and normalizing each column in $X$ or not. Our analysis implies that over-fitting can cause partly inconsistent results seen in previous studies which used the arbitrary flux ratios (Bailey et al. 2009; Blondin et al. 2011; Silverman et al. 2012).

Our conclusion is inconsistent with that reported by Silverman et al. (2012) although the both samples are obtained from the Berkeley supernova database with the common data selection. The model selection method is also common: Following Blondin et al. (2011), Silverman et al. (2012) performed 10-fold cross-validation, and calculated the mean and standard error of 10 weighted rootmean squares (wRMS) of residuals. They measured the significance of the improvement of the model with the mean wRMS and its standard error. They found that 


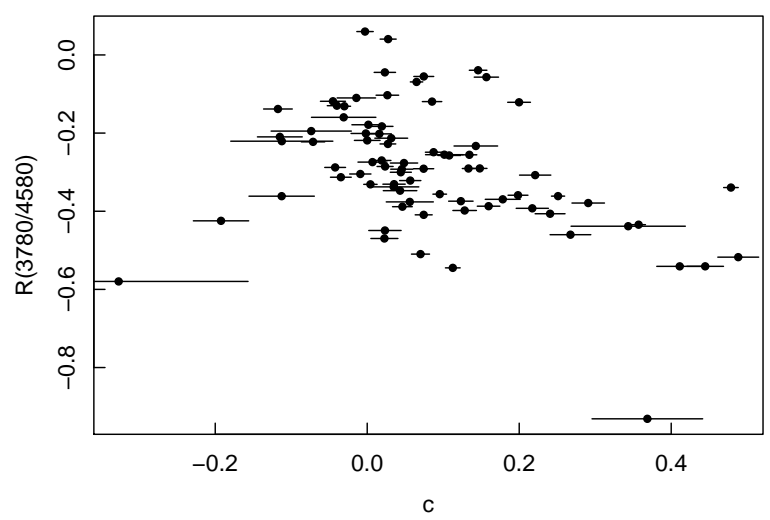

Fig. 7. $\mathcal{R}^{c}(3780 / 4580)$ of our sample against $c$

the model with $c, x_{1}$, and $\mathcal{R}^{c}(3780 / 4580)$ improves the prediction error by a level of $1.7 \sigma$ compared with the classical one with $c$ and $x_{1}$. This flux ratio is also detected as an explanatory variable in our Model 1 , while it is not in the other models of $M_{B}$ (see table 1). The wavelength of $3780 \AA$ corresponds to the mid-point of Ca II $\mathrm{H} \& \mathrm{~K}$, and $4580 \AA$ to the border between the Mg II and Fe II complexes. Figure 7 shows $\mathcal{R}^{c}(3780 / 4580)$ of our sample against $c$. Those two variables exhibit a weak anticorrelation, as can be seen in this figure. Our result that $\mathcal{R}^{c}(3780 / 4580)$ is detected in Model 1 and not in Models 2 and 3 can be explained by this anticorrelation.

In principle, the spectral data, that is, the values of the flux density and their ratios, could be important explanatory variables of $M_{B}$. The interstellar extinction is definitely the most important variable. As well as the color parameter, $c$, the continuum flux of the total-flux normalized spectra, that is, $\boldsymbol{f}_{\text {tot }}$ could be an indicator of the extinction. Indeed, $f_{\text {tot }}(6373)$ has a relatively large coefficient in Model 1. Our analysis suggests that $c$ is a better variable rather than $f_{\text {tot }}(6373)$ and other normalized fluxes. This is probably because of the uncertainty of measurements and observation epochs. In general, the flux calibration of spectra has larger errors than the differential photometry. Moreover, the observation epochs of spectra are different from one object to another in our sample. The color parameter, $c$, is based on differential photometry, and corrected to the color at maximum. A similar situation is also expected in the light-curve width, $x_{1}$. Mazzali et al. (2001) propose that $x_{1}$, or decline rate, so-called, $\Delta m_{15}$ is a function of the amount of ${ }^{56} \mathrm{Ni}$ produced in SNe. The absorption line variables are also possible indicators of the amount of synthesized elements in SNe. Indeed, the correlation between $x_{1}$ and the strength of the Si II lines was confirmed in Model 4. Our method selected not the Si II strength, but $x_{1}$ probably because of the small measurement error in $x_{1}$ for the amount of synthesized elements. In our analysis, the best set of the explanatory variables is $c$ and $x_{1}$, while it is trivial that our result does not imply a physical causal relationship between $M_{B}$ and those two variables.
It is possible that, in future, the increasing number of samples revises the model having a better generalization error by finding additional or alternative explanatory variables compared with the model in this paper. It may also be meaningful to add variables which were not used in this paper, such as those about host galaxies of SNe Ia (Sullivan et al. 2010; Pan et al. 2015). In any of these cases, our proposed method offers a framework for finding an appropriate set of explanatory variables of $M_{B}$ even in the case that the number of samples is smaller than the number of variables. A possible extension of the model may be to include the measurement errors of explanatory variables. As can be seen in equation (2), our method does not include the errors, while errors are expected to be large in several variables, for example, flux ratios of low fluxes. Bailey et al. 2009, proposing the flux ratio, $\mathcal{R}(642 \mathrm{~nm} / 443 \mathrm{~nm})$, as a good explanatory variable of $M_{B}$, claimed that the spectral slope need to be calibrated with very small errors for their model with the flux ratio. The instrument that they used was SNIFS (SuperNova Integral Field Spectrograph), which was developed to perform fluxcalibration with a high accuracy (Aldering et al. 2002). On the other hand, the instruments which were used in Blondin et al. (2011) and Silverman et al. (2012) were standard slit spectrographs. It is possible that the lack of detection of $\mathcal{R}(642 \mathrm{~nm} / 443 \mathrm{~nm})$ in our analysis is due to large errors of the flux ratios in our data sample from Silverman et al. (2012). A better model including the errors might be provided by a Bayesian approach in which the error is included into the model as prior probability distributions.

We would like to thank Drs. J. M. Silverman and A. V. Filippenko for providing the Berkeley supernova database. We also appreciate comments and suggestions from anonymous referee. This work was supported by JSPS KAKENHI Grant Number 25120007, 25120008, and 26800100. The work by K.M. is partly supported by WPI Initiative, MEXT, Japan.

\section{References}

Aldering, G., Adam, G., Antilogus, P., Astier, P., Bacon, R., Bongard, S., Bonnaud, C., Copin, Y., et al. 2002, in Survey and Other Telescope Technologies and Discoveries, ed. J. A. Tyson, \& S. Wolff Vol. 4836 of Society of Photo-Optical Instrumentation Engineers (SPIE) Conference Series, pp $61-72$

Arsenijevic, V., Fabbro, S., Mourão, A. M., \& Rica da Silva, A. J. 2008, A\&A, 492, 535

Bailey, S., Aldering, G., Antilogus, P., Aragon, C., Baltay, C., Bongard, S., Buton, C., Childress, M., et al. 2009, A\&A, 500, L17

Blondin, S., Mandel, K. S., \& Kirshner, R. P. 2011, A\&A, 526, A 81

Conley, A., Carlberg, R. G., Guy, J., Howell, D. A., Jha, S., Riess, A. G., \& Sullivan, M. 2007, ApJL, 664, L13

Foley, R. J., Challis, P. J., Chornock, R., Ganeshalingam, M., Li, W., Marion, G. H., Morrell, N. I., Pignata, G., et al. 2013, ApJ, 767, 57

Foley, R. J. \& Kasen, D. 2011, ApJ, 729, 55 
Guy, J., Astier, P., Baumont, S., Hardin, D., Pain, R., Regnault, N., Basa, S., Carlberg, R. G., et al. 2007, A\&A, 466, 11

Hachinger, S., Mazzali, P. A., \& Benetti, S. 2006, MNRAS, 370,299

Mazzali, P. A., Nomoto, K., Cappellaro, E., Nakamura, T., Umeda, H., \& Iwamoto, K. 2001, ApJ, 547, 988

Pan, Y.-C., Sullivan, M., Maguire, K., Gal-Yam, A., Hook, I. M., Howell, D. A., Nugent, P. E., \& Mazzali, P. A. 2015, MNRAS, 446, 354

Peek, J. E. G. \& Graves, G. J. 2010, ApJ, 719, 415

Phillips, M. M. 1993, ApJL, 413, L105

Schlegel, D. J., Finkbeiner, D. P., \& Davis, M. 1998, ApJ, 500, 525

Silverman, J. M., Ganeshalingam, M., Li, W., \& Filippenko, A. V. 2012, MNRAS, 425, 1889

Sullivan, M., Conley, A., Howell, D. A., Neill, J. D., Astier, P., Balland, C., Basa, S., Carlberg, R. G., et al. 2010, MNRAS, 406, 782

Tibshirani, R. 1996, Journal of the Royal Statistical Society. Series B (Methodological), 58, 267 